\title{
Short communication: Circulating fatty acylcarnitines are elevated in overweight periparturient dairy cows in association with sphingolipid biomarkers of insulin resistance
}

\author{
J. E. Rico, ${ }^{*}$ Y. Zang, ${ }^{*}$ N. J. Haughey,† A. G. Rius, $\ddagger$ and J. W. McFadden ${ }^{* 1,2}$ \\ *Division of Animal and Nutritional Sciences, West Virginia University, Morgantown 26505 \\ †Department of Neurology, Johns Hopkins University School of Medicine, Baltimore, MD 21287 \\ ‡Department of Animal Science, University of Tennessee, Knoxville 37996
}

\begin{abstract}
Defects in mitochondrial fatty acid processing are associated with the development of fatty liver disease, inflammation, and insulin resistance in overweight nonruminants. Surplus fatty acids (FA) and defects in FA oxidation favor the accumulation of fatty acylcarnitines (FAC) and the sphingolipid ceramide. Moreover, elevated circulating FAC and ceramide concentrations are inversely related to insulin action. Because we have previously demonstrated that plasma ceramide levels increase during the transition from gestation to lactation, our aim was to determine whether changes in plasma medium- and long-chain FAC levels are related to circulating FA and sphingolipids in peripartal dairy cows. We hypothesized that plasma FAC levels would be higher in overweight cows experiencing increased lipolysis, and that FAC levels would be positively associated with elevations in plasma ceramides. Twenty-one multiparous Holstein cows were grouped according to body condition score (BCS) at d -30 prepartum as lean (BCS $<3.0 ; n=10)$ or overweight $(\mathrm{BCS}>4.0 ; \mathrm{n}$ $=11$ ). Blood was collected at $\mathrm{d}-30,-15,-7$, and 4 , relative to parturition. Circulating FAC and ceramide levels were determined using liquid chromatography and tandem mass spectrometry. To investigate the potential contributions of sphingomyelin (SM) hydrolysis to ceramide accrual, we also determined plasma SM levels during the peripartum period. Data were analyzed under a mixed model with the fixed effects of adiposity and time, and the random effect of cow. Relative to lean cows, overweight cows had elevated FAC during the transition from gestation to lactation. Circulating FAC levels were positively associated
\end{abstract}

\footnotetext{
Received May 15, 2017.

Accepted September 16, 2017.

${ }^{1}$ Corresponding author: jwm43@cornell.edu

${ }^{2}$ Present address: Cornell University, 264 Morrison Hall, Ithaca, NY 14853.
}

with FA, ceramide, and dihydro-SM levels. Although circulating FAC levels increased in all cows during the peripartum, enhanced prepartum adiposity contributed to a greater rise in plasma FA and FAC. Our results support on-going efforts to determine whether altered mitochondrial FA processing promotes the accumulation of the insulin resistance biomarker ceramide in blood and liver.

Key words: ceramide, fatty acylcarnitine, lipotoxicity, sphingomyelin

\section{Short Communication}

Elevated genetic merit for milk production imposes heightened energy requirements for modern dairy cows, resulting in severe negative energy balance that is prolonged over early lactation (Coppock and Wilks, 1991; Grummer et al., 2004). Although lipomobilization from adipose tissue constitutes a key response for maintaining energy homeostasis during early lactation, excessive levels of circulating free fatty acids (FA) can cause metabolic stress and increase the propensity of cows to develop peripartal metabolic diseases (e.g., ketosis and fatty liver; Dechow et al., 2004; Ospina et al., 2010). Moreover, rampant lipolysis is directly associated with gestational adiposity (Kim and Suh, 2003; Rico et al., 2015 ) and the extent of adipose tissue-specific insulin antagonism during peripartum (Zachut et al., 2013). Consequently, body fat accumulation is a risk factor for metabolic disease in nonruminants (Grundy, 2004), as well as the hyperlipidemic peripartal dairy cow (Roche et al., 2009). Furthermore, overweight individuals exhibit increased sedentary time, which increases their risk for developing insulin resistance (Hamilton et al., 2007), and low physical activity is positively associated with health disorders in dairy cows transitioning from gestation to lactation (Edwards and Tozer, 2004).

Although the association between elevated circulating FA concentrations and metabolic impairment is well established (i.e., lipotoxicity; DeFronzo, 2004; 
Summers, 2006; Ospina et al., 2010), our understanding of the factors underlying the propensity for peripartal disease in hyperlipidemic overweight cows remains incomplete. Studies in rodents and humans indicate that alterations in FA processing (e.g., catabolism vs. anabolism) can play an important role in the onset of lipid-associated metabolic dysfunction. In dairy cows, circulating FA levels increase immediately postpartum, particularly in overweight cows (Rico et al., 2015), and omics has revealed peripartal alterations in lipid metabolism (Hailemariam et al., 2014; Imhasly et al., 2014; Rico et al., 2017). During this critical period, the capacity to completely oxidize FA (i.e., palmitate) to $\mathrm{CO}_{2}$ is reduced, whereas incomplete $\beta$-oxidation to acid-soluble products (i.e., ketones and TCA cycle intermediates) is enhanced (Litherland et al., 2011). Moreover, these conditions favor the hepatic synthesis of esterified products, such as triacylglycerols (TAG). In nonruminants, increased hepatic FA influx also promotes the accumulation of fatty acylcarnitines (FAC; Schooneman et al., 2013). Fatty acylcarnitines are FA esters formed when fatty acyl-CoA are shuttled into the mitochondrion for $\beta$-oxidation, a process facilitated by carnitine palmitoyltransferase 1 (McGarry and Brown, 1997). Because FAC are formed when activated FA are targeted for oxidation, they are regarded as potential indicators of defects in FA processing (Adams et al., 2009; Ramos-Roman et al., 2012). The hepatic accumulation of FAC can promote their accrual in circulation, a process potentially facilitated by organic cation/ carnitine transporter 2 (Schooneman et al., 2013). Indeed, hepatic FA influx is accompanied by elevations in circulating FAC in obese subjects diagnosed with type 2 diabetes (Mihalik et al., 2010), as well as in individuals with nonalcoholic fatty liver disease (Kalhan et al., 2011). In dairy cows, short-chain propionyl-carnitine has been reported to be elevated in cows with compromised health (i.e., mastitis, metritis, laminitis, and retained placenta; Hailemariam et al., 2014). However, we recognize that elevations in long-chain FAC can occur in mid-lactation cows with healthy liver function (Huber et al., 2016).

Although hepatic lipid accumulation is characterized by TAG deposition and FAC accumulation, intracellular FA can be partitioned to other metabolic fates, including the synthesis of the insulin resistance biomarker ceramide (Haus et al., 2009; Watt et al., 2012; Schooneman et al., 2013). We recently reported that plasma ceramides are positively associated with circulating FA in overweight dairy cows during the peripartum period (Rico et al., 2015, 2017). We postulated that ceramide accrual may be due to enhanced de novo ceramide synthesis from FA, as observed in hyperlipidemic nonruminants with fatty liver disease (Raichur et al., 2014). Al- ternatively, ceramide generation from proinflammatory tumor necrosis factor- $\alpha$-provoked sphingomyelin (SM) hydrolysis may be the cause (Peraldi et al., 1996). To further characterize the potential relationship between FA oxidative metabolism and ceramide metabolism, our goal was to characterize changes in plasma FAC levels in peripartal lean and overweight dairy cows, and to delineate their relationship to FA and sphingolipid supply. We hypothesized that plasma FAC levels would increase in overweight cows experiencing enhanced lipolysis, and FAC levels would be positively associated with elevations in plasma ceramides.

To test our hypothesis, an experiment was completed at Dovan Farms (Berlin, PA), West Virginia University Agricultural Research and Education Partner, and a 700-Holstein cow commercial dairy farm (Berlin, PA). Nonlactating, pregnant, multiparous Holstein cows (n $=21$ ) were enrolled in the study $45 \mathrm{~d}$ before expected parturition and allocated to 1 of 2 groups according to their adiposity at $\mathrm{d}-30$ relative to calving, as either lean (BCS $=2.9 \pm 0.22, \mathrm{n}=10)$ or overweight (i.e., overconditioned; $\mathrm{BCS}=4.1 \pm 0.10, \mathrm{n}=11$ ), as reported in Rico et al. (2015). Parity was not different between BCS groups $(P>0.9)$, averaging $3.5 \pm$ 0.82 and $3.45 \pm 1.08$ for lean and overweight cows, respectively. Experimental procedures were approved by the West Virginia University Institutional Animal Care and Use Committee.

Diets were formulated to meet nutrient recommendations and were primarily composed of corn silage and grass haylage, as described previously (Rico et al., 2015). Contents of NDF, CP, and starch were 48, 12.2 , and $15 \%$ for the prepartum diet, and 33.4, 16.5, and $25 \%$ for the lactation diet, respectively. Animals were fed for ad libitum intake with free access to water. Weekly TMR samples were analyzed for nutrient composition by near-infrared spectroscopy (Cumberland Valley, Cumberland, MD; AOAC International, 1995; method 989.03). Body weights and BCS (1.0 to 5.0 scale; Wildman et al., 1982) were recorded weekly. Three trained investigators independently recorded BCS for all cows and data were averaged for each cow at $\mathrm{d}-30,-15,-7,4,14$, and 21 relative to expected parturition. Cows were fitted with collar-mounted activity meters for hourly activity data collection, transmitted to an antenna, and recorded using ALPRO software (DeLaval, Kansas City, MO). Prior to morning feeding, blood samples $(10 \mathrm{~mL})$ were collected by coccygeal venipuncture at $\mathrm{d}-30,-15,-7$, and 4 relative to expected parturition. Blood was kept on ice for 30 min until centrifugation at $3,400 \times g$ for $10 \mathrm{~min}$ at $4^{\circ} \mathrm{C}$. Plasma containing EDTA was removed, immediately snap-frozen in liquid nitrogen, and stored at $-80^{\circ} \mathrm{C}$. Postpartum blood sampling at d 4 was selected because 
this time reflects maximum FA mobilization (Janovick et al., 2011).

Plasma concentrations of glucose and FA were determined by enzymatic methods and plasma insulin was measured using immunoassay, as previously described (Rico et al., 2015). Intra- and interassay coefficients of variation were 3.8 and $3.0 \%, 2.6$ and $7.7 \%$, and 3.7 and $16.6 \%$, for glucose, FA, and insulin, respectively. Targeted lipidomic analyses were conducted using liquid chromatography and tandem mass spectrometry, as previously described (Haughey et al., 2004; Rico et al., 2015). Briefly, a modified Bligh and Dyer procedure was used to obtain plasma lipid extracts, dried under nitrogen, resuspended in methanol, introduced into an HPLC (PerkinElmer, Boston, MA), and injected into an electrospray ion source coupled with a triple quadrupole mass spectrometer (API3000; AB Sciex Inc., Thornhill, ON, Canada). Fold-change of all plasma metabolites was calculated as change relative to values of lean cows at $\mathrm{d}-30$ relative to parturition.

All data were analyzed as repeated measures over time relative to parturition using the MIXED procedure of SAS (SAS 9.3; SAS Institute Inc., Cary, NC). The model included the random effect of cow nested within BCS and the fixed effects of BCS, day relative to parturition, and their interaction. The most appropriate covariance structure for the repeated-measures analysis was selected as previously described (Rico et al., 2015). The SLICE option of SAS was used to compare treatment differences at individual time points. Parametric Pearson correlations were calculated to determine associations between variables, and regression analysis was performed using the REG procedure of SAS. The full model included the linear and quadratic effects of each predictor. Studentized residual values $>3.0$ or $<-3.0$ were considered outliers and removed from the analysis (typically 1 per response variable). All results are expressed as least squares means and their standard errors, unless stated otherwise. Significance was declared at $P<0.05$ and tendencies at $P<0.10$.

Although BCS groups used in our study did not reflect the average distribution of BCS commonly observed in dairy herds, our adiposity groups allowed for the evaluation of metabolism of cows with a low and high propensity for increased lipolysis and, thus, contrasting disease risk. As our research group has previously described (Rico et al., 2015), overweight cows experienced accelerated fat mass loss relative to lean cows ( -22 vs. $-13 \%$, respectively; $P<0.01$ ) during the course of peripartum (d -30 to 21 ). Similarly, and suggestive of prepartum insulin intolerance, overweight cows had elevated concentrations of glucose and insulin compared to lean cows, the latter being linearly related to adiposity (Supplemental Figure S1A-C; https://doi .org/10.3168/jds.2017-13171). The reductions in plasma insulin occurred concomitantly with progressive elevations of circulating C24:0- and total ceramide, which were higher in overweight cows by d 4 postpartum (Supplemental Figure S1D-E). From multiple observations, C24:0-ceramide has emerged as a putative biomarker for insulin antagonism in hyperlipidemic dairy cows transitioning from gestation to lactation (Rico et al., 2015, 2017), as well as in cows fed palmitic acid during established lactation (Rico et al., 2016).

Circulating FA concentrations increased more than 4 fold during the peripartum period in lean and overweight cows (Figure 1A). Consistent with the prepartum onset of insulin resistance, overweight cows had elevated FA levels relative to lean as early as d -15 prepartum. Our mass spectrometry analysis revealed plasma changes in medium- and long-chain FAC, including myristoyl-, palmitoyl-, stearoyl-, and arachidonyl-carnitine (C14:0-, C16:0-, C18:0-, and C20:0-FAC, respectively; Figure 1B-E). Similar to the FA profile of circulating FA during the transition period (Contreras et al., 2010), where lipolysis-derived palmitic and stearic acid constitute the major circulating FA, we found that $\mathrm{C} 16: 0$ - and $\mathrm{C} 18: 0-\mathrm{FAC}$ predominated in plasma (32 and $48 \%$ of detected FAC, respectively). Moreover, all detected plasma FAC increased from d -30 to -7 , and the elevations were more pronounced in overweight animals. Of note, C16:0- and C18:0-FAC increased by 3.6 and 2.6 fold, respectively, in overweight cows from $\mathrm{d}-30$ to $-7(P<0.05)$; similar changes were observed for C14:0- and C20:0-FAC $(P<0.05)$. All plasma FAC levels decreased at d 4 postpartum $(P<0.001)$. Interestingly, these responses may be due to reduced plasma carnitine concentrations during early lactation (Schlegel et al., 2012) or downregulation of carnitine palmitoyltransferase 1 (Xu et al., 2011). Overall, FAC levels were linearly associated with FA during the transition from gestation to lactation (Figure 1F). Further regression analysis revealed that plasma FAC were positively associated with circulating total ceramide (Figure 1G), suggesting that FAC and de novo ceramide synthesis occurred simultaneously. Notably, the correlation was strongest for C24:0-ceramide (Figure $1 \mathrm{H}$ ), a highly abundant ceramide found in bovine plasma and liver, and associated with the severity of peripheral insulin intolerance during peripartum and established lactation (Rico et al., 2015, 2016, 2017).

The sphingolipids ceramide and SM are interconnected in their degradation and synthesis pathways (Spiegel and Milstien, 2003). For example, dihydro-SM (DHSM) are derived from intermediates of the de novo ceramide synthesis pathway; however, their role in controlling insulin action has not been explored. Although our lipidomics profiling was limited to 3 DHSM 
- Lean OOverweight
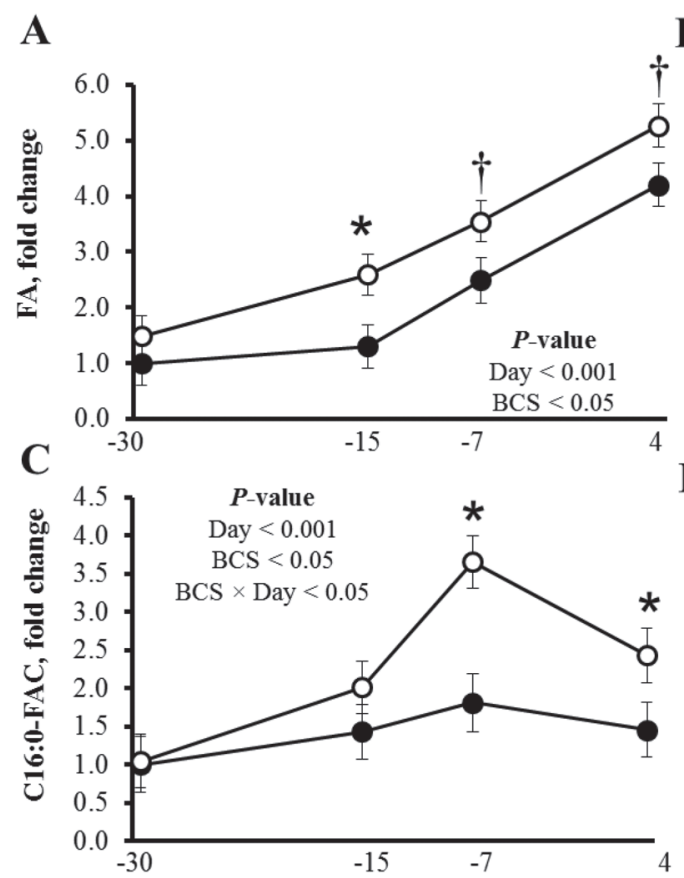

B

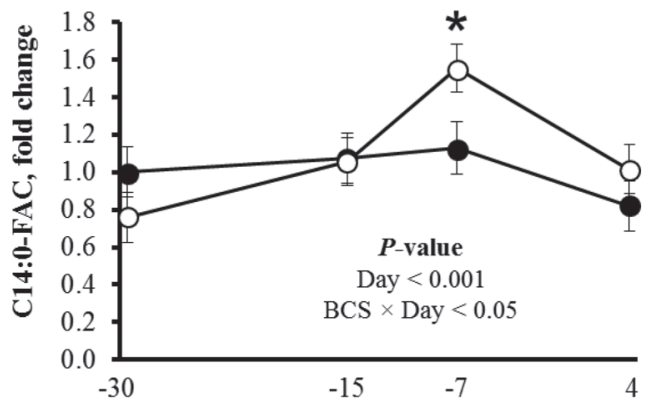

D

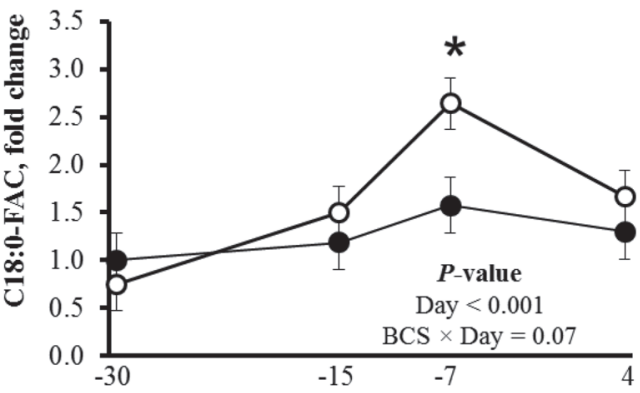

E

F

Day relative to parturition

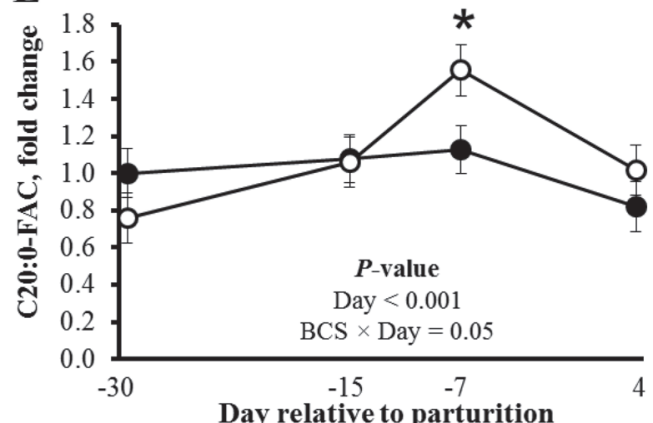

G
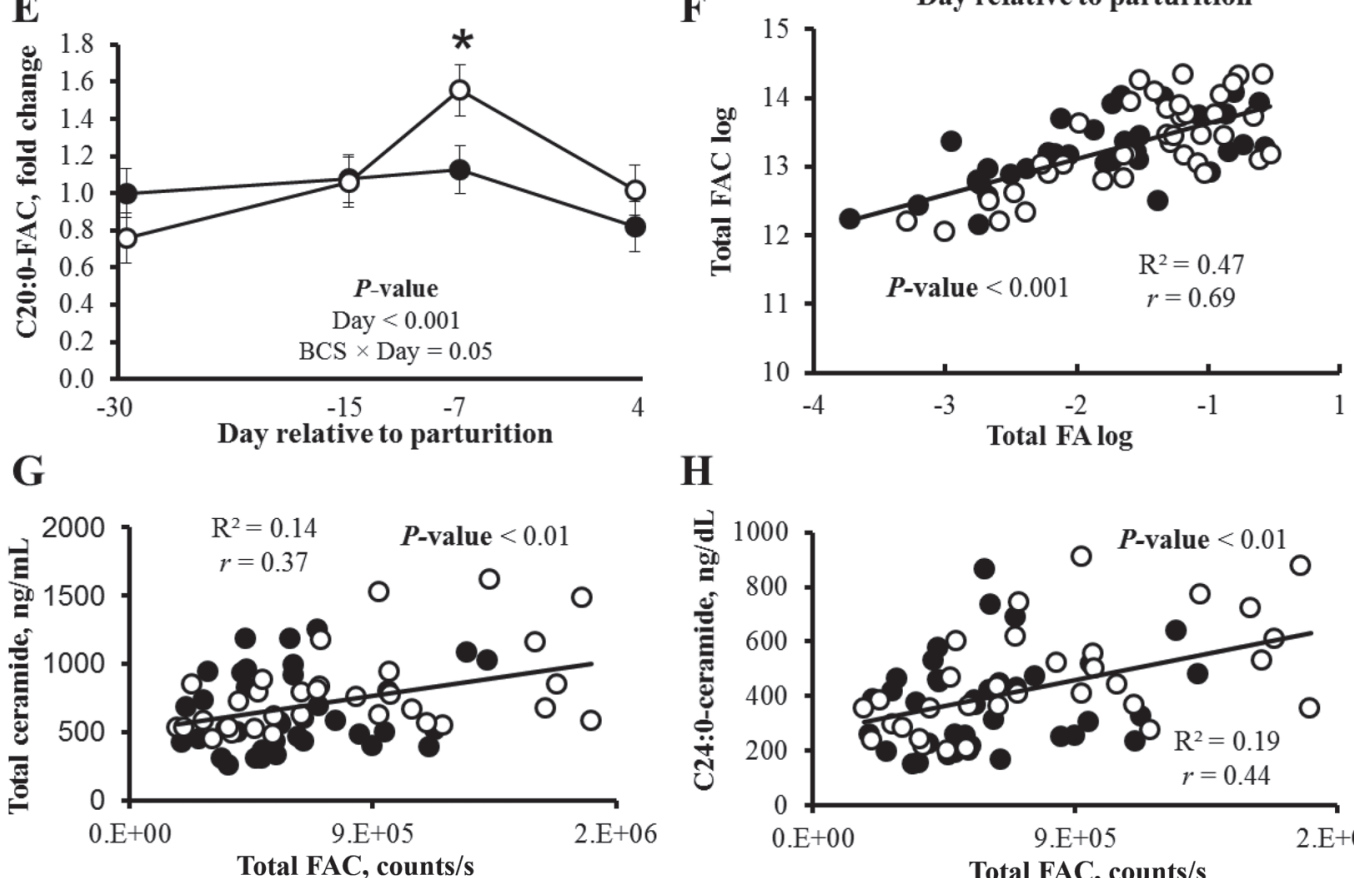

H

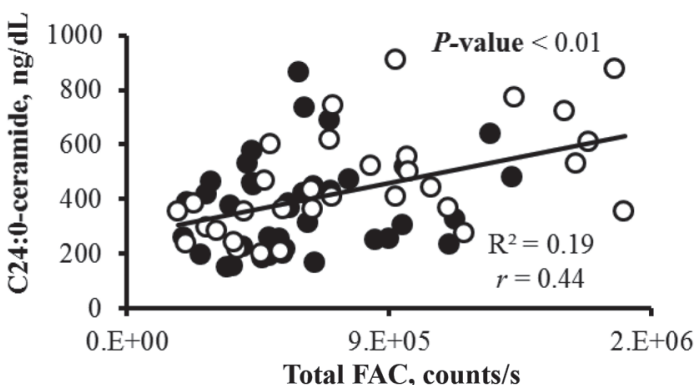

Figure 1. Circulating fatty acylcarnitines (FAC) are elevated in overweight cows and are associated with fatty acids (FA) and ceramide levels. Plasma fold change in (A) FA, (B) C14:0-, (C) C16:0-, (D) C18:0-, and (E) C20:0-FAC. Linear regression analysis of (F) plasma FA and FAC, $(\mathrm{G})$ total ceramide and FAC, and $(\mathrm{H}) \mathrm{C} 24: 0$-ceramide and FAC in lean and overweight periparturient dairy cows. Data are shown as LSM and SE. ${ }^{*} P<0.05 ; \dagger P<0.10$. Fold change was calculated as change relative to lean at $\mathrm{d}-30$.

moieties (i.e., C16:0-, C18:0-, and C20:0-DSM; Figure 2A; Supplemental Figure S2A-B, https://doi.org/10 .3168/jds.2017-13171), we observed elevated circulating C20:0-DHSM in overweight cows (Figure 2A), a
DHSM moiety that displayed a moderately high linear association to plasma total FAC levels (Figure 2B). Furthermore, total DHSM (sum of C16:0-, C18:0-, and C20:0-DHSM) showed moderate associations with long- 
chain ceramides $(\mathrm{r}=0.55,0.59$, and 0.58 for $\mathrm{C} 20: 0-$, C22:0-, and C24:0-ceramide, respectively; $P<0.001$ ).

Considering that ceramides can be generated from dif- ferent cellular pathways and that DHSM are generated from de novo dihydro-ceramides (van Helvoort, et al., 1997; Spiegel and Milstien, 2003), the possibility of us-

- Lean OOverweight
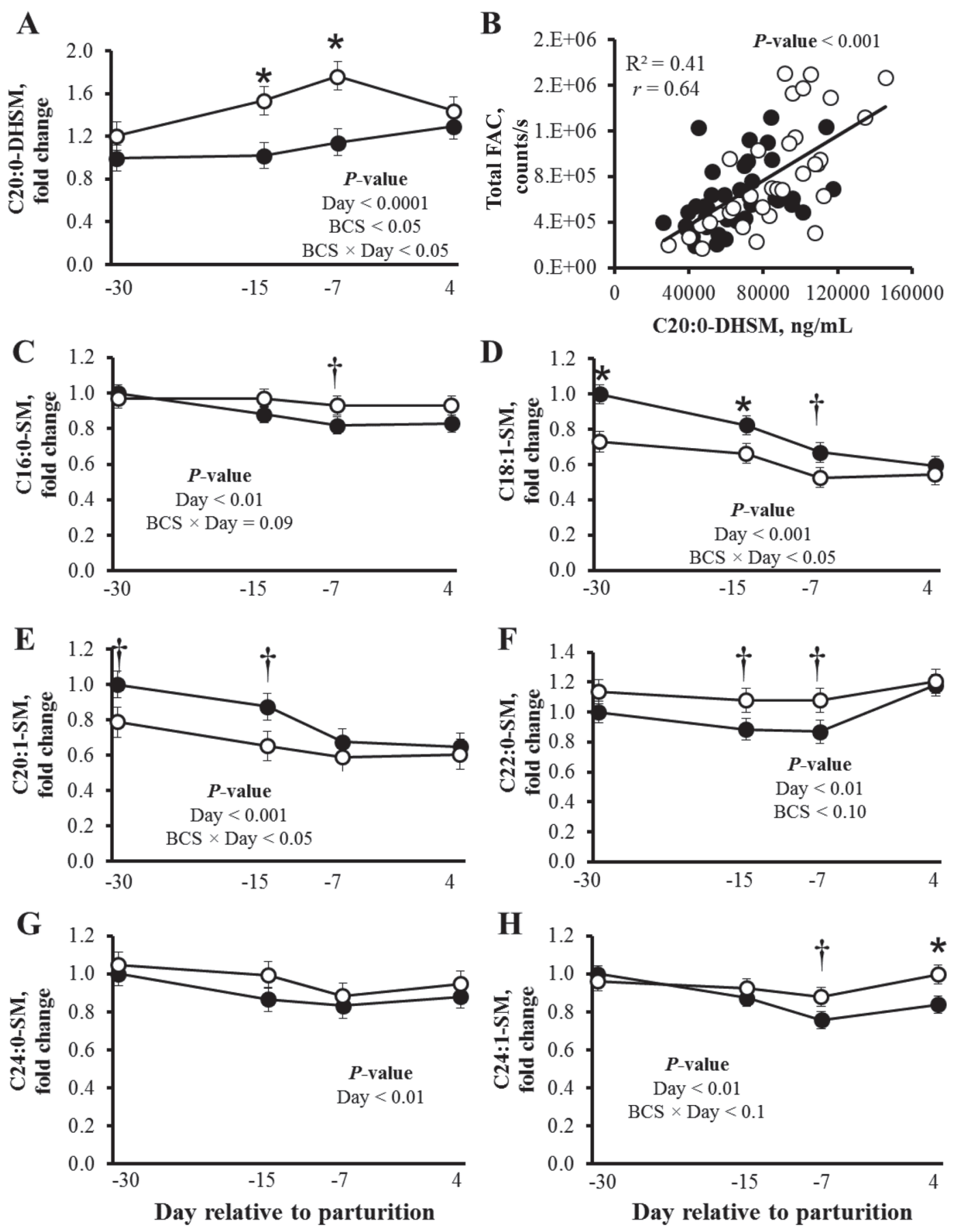

Figure 2. C20:0-Dihydrosphingomyelin (DHSM) is elevated in overweight cows and is linearly associated with circulating fatty acylcarnitines (FAC) during the transition from late pregnancy to early lactation. (A) Peripartal changes in plasma C20:0-DHSM, and (B) regression analysis of plasma FAC and C20:0-DHSM. Changes in plasma (C) C16:0-, (D) C18:1-, (E) C20:1-, (F) C22:0-, (G) C24:0-, and (H) C24:1-sphingomyelin $(\mathrm{SM})$ in lean and overweight dairy cows from late pregnancy to early lactation. Data are shown as LSM and SE of fold-change. ${ }^{*} P<0.05 ; \dagger P$ $<0.10$. Fold change was calculated as change relative to lean at $\mathrm{d}-30$. 
ing DHSM monitoring as a proxy for de novo ceramide synthesis should be further explored.

Alternative to de novo ceramide synthesis from a SFA substrate, SM hydrolysis may contribute to ceramide synthesis, a process facilitated by the proinflammatory activation of sphingomyelinase (Peraldi et al., 1996). Indeed, increased expression of genes involved in FA partitioning and macrophage recruitment and inflammation are observed in human subjects with fatty liver (Westerbacka et al., 2007); notably, these conditions are common for postpartum dairy cows with metabolic stress (Sordillo and Raphael, 2013). Validating our recent report (Rico et al., 2017), we observed consistent peripartal reductions in several circulating SM, including the major moieties C16:0-, C18:1-, and C20:1 -SM (Figure 2C-H), as well as total SM (Supplemental Figure S2C, https://doi.org/10.3168/jds.2017-13171; $P$ $<0.001)$. Moreover, the plasma levels of the majority of circulating SM were inversely associated with ceramide (Supplemental Figure S2D; $P<0.05$ ). Some SM moieties tended to be $(P<0.1)$ or were significantly elevated $(P<0.05)$ in overweight cows (e.g., C16:0-, C20:0-, and C24:1-SM), similar to observations of elevated SM in plasma collected from young obese humans displaying fatty liver and insulin resistance (Hanamatsu et al., 2014). In contrast, C18:1- and C20:1-SM were elevated in lean cows prepartum $(P<0.05)$, further validating our previous observations (Rico et al., 2017). Lastly, a moderate negative association between circulating FAC levels and C18:1- and C20:1-SM was detected (r $=-0.27 ; P<0.05)$. Although evidence suggests that C16:0- and C24:0-ceramide can inhibit insulin signaling (Turpin et al., 2014; Boon et al., 2013), no mechanistic data are available to suggest an effect of specific SM on insulin sensitivity, thus limiting our ability to determine the significance of the changes observed for several of the sphingolipid moieties.

Reduced physical activity is associated with weight gain and insulin resistance in humans (Hu et al., 2001) through metabolic processes that can result in reduced mitochondrial citric acid cycle activity and FAC accumulation (Koves et al., 2008; Ribel-Madsen et al., 2016). Increasing physical activity reduces ceramide concentrations in rodent skeletal muscle tissue, as well as in plasma collected from obese type 2 diabetic humans (Dobrzyń and Górski, 2002; Kasumov et al., 2015). Despite a numerical increase in physical activity in overweight cows over a 24 -h period, our analysis of peripartal activity in lean and overweight cows detected no effect of adiposity (Supplemental Figure S3; https://doi.org/10.3168/jds.2017-13171) and no associations between activity and circulating FAC. However, low and moderate associations were observed between plasma sphingolipids and daily activity. For example,
C24:0-ceramide, C20:0-DHSM, and C24:1-SM, all of which were elevated in overweight cows, were inversely associated with daily activity $(\mathrm{r}=-0.22$ to $-0.39 ; P$ $=0.07$ to $<0.001)$. These preliminary data suggest that mechanisms leading to sphingolipid accumulation may be related to physical activity even in the absence of changes in circulating FAC. This would be in agreement with reports demonstrating that increased energy expenditure, which promotes FA oxidation, leads to suppression of ceramide synthesis (Holland et al., 2013).

The observed prepartum elevation of circulating FAC in overweight cows is consistent with previous reports demonstrating that lipid-induced mitochondrial stress involves incomplete $\beta$-oxidation and FAC spillover into circulation (Koves et al., 2008; Mihalik et al., 2010), which in turn can be reflective of impaired insulin sensitivity (Adams et al., 2009; Mihalik et al., 2010). In addition to this associative role, recent evidence suggests that FAC can also directly antagonize insulin signaling at the level of protein kinase B phosphorylation (Aguer et al., 2015). Although we did not directly measure insulin sensitivity in our study, we did observe elevated plasma glucose and FA concentrations in overweight cows despite insulin concentrations being $\sim 50 \%$ higher relative to lean cows, an indication of impaired insulin action during late gestation. Moreover, the rise in circulating FAC supply developed in parallel with ceramide accrual thus supporting recent findings that have established ceramide (e.g., C24:0-ceramide) as a biomarker for insulin antagonism in dairy cattle (Rico et al., 2015, 2016, 2017) and diabetic humans (Haus et al., 2009). The mechanisms that control the partitioning of lipolysis-derived FA toward hepatic ceramide synthesis may involve the inactivation of mitochondrial electron transport (Raichur et al., 2014), which in turn increases FA substrate supply for serine palmitoyltransferase 1 (Watt et al., 2012). Although not measured in the current study, we have recently demonstrated that the hepatic C24:0-ceramide concentration increase in overweight cows transitioning from gestation to lactation (Rico et al., 2017), a response that may further decelerate hepatic $\beta$-oxidation and exacerbate TAG accumulation (Raichur et al., 2014; Hla and Kolesnick, 2014). In addition to the de novo ceramide synthesis pathway as a likely contributor to liver ceramide secretion, the observed decreases in SM levels in the present study is in line with a potential role for activated sphingomyelinase. In support, acid sphingomyelinase activity is upregulated in subjects experiencing nonalcoholic fatty liver disease and ceramide accumulation (Grammatikos et al., 2014), and obesity-associated inflammation can promote the transformation of SM to ceramide (Peraldi et al., 1996). The relative contributions of the bovine de novo synthesis and SM hydrolysis pathways toward 
the early lactation rise in circulating ceramide should be characterized in the future.

Our results demonstrated that FAC accumulation exists in all cows during the peripartum period, but it is further increased in cows with higher adiposity. The simultaneous increases in ceramide and DHSM levels suggest that excess FA that are not oxidized are, in turn, diverted toward de novo ceramide synthesis and subsequently secreted into circulation, where they may antagonize insulin action at target tissues. Additionally, ceramide synthesis may also be attributed to SM hydrolysis. These findings support the emerging concept that alterations in FA processing facilitate the synthesis of lipid mediators of metabolic disease in dairy cattle.

\section{ACKNOWLEDGMENTS}

This work was supported by the USDA Northeast Sustainable Agriculture Research and Education Program (\#GNE13-067) and the USDA Agriculture and Food Research Initiative (AFRI) Competitive Grant (\#WVA00902). We thank DeLaval (Kansas City, MO) for providing the activometers and the VanGilder family at DoVan Farms for their participation.

\section{REFERENCES}

Adams, S. H., L. C. Hoppel, K. H. Lok, L. Zhao, S. W. Wong, P. E. Minkler, D. H. Hwang, J. W. Newman, and W. T. Garvey. 2009. Plasma acylcarnitine profiles suggest incomplete long-chain fatty acid $\beta$-oxidation and altered tricarboxylic acid cycle activity in type 2 diabetic African-American women. J. Nutr. 139:1073-1081.

Aguer, C., C. S. McCoin, T. A. Knotts, A. B. Thrush, K. Ono-Moore, R. McPherson, R. Dent, D. H. Hwang, S. H. Adams, and M. E. Harper. 2015. Acylcarnitines: Potential implications for skeletal muscle insulin resistance. FASEB J. 29:336-345.

AOAC International. 1995. Official Methods of Analysis. 16th ed. AOAC International, Arlington, VA.

Boon, J., A. J. Hoy, R. Stark, R. D. Brown, R. C. Meex, D. C. Henstridge, S. Schenk, P. J. Meikle, J. F. Horowitz, B. A. Kingwell, C. R. Bruce, and M. J. Watt. 2013. Ceramides contained in LDL are elevated in type 2 diabetes and promote inflammation and skeletal muscle insulin resistance. Diabetes 62:401-410.

Contreras, G. A., N. J. O'boyle, T. H. Herdt, and L. M. Sordillo. 2010 Lipomobilization in periparturient dairy cows influences the composition of plasma nonesterified fatty acids and leukocyte phospholipid fatty acids. J. Dairy Sci. 93:2508-2516.

Coppock, C. E., and D. L. Wilks. 1991. Supplemental fat in highenergy rations for lactating cows: Effects on intake, digestion, milk yield, and composition. J. Anim. Sci. 69:3826-3837.

Dechow, C. D., G. W. Rogers, L. Klei, and T. J. Lawlor. 2004. Heritability and correlations for body condition score and dairy form within and across lactation and age. J. Dairy Sci. 87:717-728.

DeFronzo, R. A. 2004. Dysfunctional fat cells, lipotoxicity and type 2 diabetes. Int. J. Clin. Pract. Suppl. 143:9-21.

Dobrzyń, A., and J. Górski. 2002. Ceramides and sphingomyelins in skeletal muscles of the rat: content and composition. Effect of prolonged exercise. Am. J. Physiol. Endocrinol. Metab. 282:E277E285.

Edwards, J. L., and P. R. Tozer. 2004. Using activity and milk yield as predictors of fresh cow disorders. J. Dairy Sci. 87:524-531.
Grammatikos, G., C. Mühle, N. Ferreiros, S. Schroeter, D. Bogdanou, S. Schwalm, G. Hintereder, J. Kornhuber, S. Zeuzem, C. Sarrazin, and J. Pfeilschifter. 2014. Serum acid Sphingomyelinase is upregulated in chronic hepatitis $\mathrm{C}$ infection and nonalcoholic fatty liver disease. Biochim. Biophys. Acta 1841:1012-1020.

Grummer, R. R., D. G. Mashek, and A. Hayirli. 2004. Dry matter intake and energy balance in the transition period. Vet. Clin. North Am. Food Anim. Pract. 20:447-470.

Grundy, S. M. 2004. Obesity, Metabolic syndrome, and cardiovascular disease. J. Clin. Endocrinol. Metab. 89:2595-2600.

Hailemariam, D., R. Mandal, F. Saleem, S. M. Dunn, D. S. Wishart, and B. N. Ametaj. 2014. Identification of predictive biomarkers of disease state in transition dairy cows. J. Dairy Sci. 97:2680-2693.

Hamilton, M. T., D. G. Hamilton, and T. W. Zderic. 2007. Role of low energy expenditure and sitting in obesity, metabolic syndrome, type 2 diabetes, and cardiovascular disease. Diabetes 56:2655-2667.

Hanamatsu, H., S. Ohnishi, S. Sakai, K. Yuyama, S. Mitsutake, H. Takeda, S. Hashino, and Y. Igarashi. 2014. Altered levels of serum sphingomyelin and ceramide containing distinct acyl chains in young obese adults. Nutr. Diabetes 4:e141.

Haughey, N. J., R. G. Cutler, A. Tamara, J. C. McArthur, D. L. Vargas, C. A. Pardo, J. Turchan, A. Nath, and M. P. Mattson. 2004. Perturbation of sphingolipid metabolism and ceramide production in HIV-dementia. Ann. Neurol. 55:257-267.

Haus, J. M., S. R. Kashyap, T. Kasumov, R. Zhang, K. R. Kelly, R. A. DeFronzo, and J. P. Kirwan. 2009. Plasma ceramides are elevated in obese subjects with type 2 diabetes and correlate with the severity of insulin resistance. Diabetes 58:337-343.

Hla, T., and R. Kolesnick. 2014. C16:0-ceramide signals insulin resistance. Cell Metab. 20:703-705.

Holland, W. L., A. C. Adams, J. T. Brozinick, H. H. Bui, Y. Miyauchi, C. M. Kusminski, S. M. Bauer, M. Wade, E. Singhal, C. C. Cheng, K. Volk, M.-S. Kuo, R. Gordillo, A. Kharitonenkov, and P. E. Scherer. 2013. An FGF-21-adiponectin-ceraide axis controls energy expenditure and insulin action in mice. Cell Metab. 17:790-797.

Hu, F. B., M. F. Leitzmann, M. J. Stampfer, G. A. Colditz, W. C. Willett, and E. B. Rimm. 2001. Physical activity and television watching in relation to risk for type 2 diabetes mellitus in men. Arch. Intern. Med. 161:1542-1548.

Huber, K., S. Dänicke, J. Rehage, H. Sauerwein, W. Otto, U. RolleKampczyk, and M. von Bergen. 2016. Metabotypes with properly functioning mitochondria and anti-inflammation predict extended productive life span in dairy cows. Sci. Rep. 6:24642.

Imhasly, S., H. Naegeli, S. Baumann, M. von Bergen, A. Luch, H. Jungnickel, S. Potratz, and C. Gerspach. 2014. Metabolomic biomarkers correlating with hepatic lipidosis in dairy cows. BMC Vet. Res. 10:122.

Janovick, N. A., Y. R. Boisclair, and J. K. Drackley. 2011. Prepartum dietary energy intake affects metabolism and health during the periparturient period in primiparous and multiparous Holstein cows. J. Dairy Sci. 94:1385-1400.

Kalhan, S. C., L. Guo, J. Edmison, S. Dasarathy, A. J. McCullough, R. W. Hanson, and M. Milburn. 2011. Plasma metabolomic profile in nonalcoholic fatty liver disease. Metabolism 60:404-413.

Kasumov, T., T. P. Solomon, C. Hwang, H. Huang, J. M. Haus, R. Zhang, and J. P. Kirwan. 2015. Improved insulin sensitivity after exercise training is linked to reduced plasma C14:0 ceramide in obesity and type 2 diabetes. Obesity (Silver Spring) 23:1414-1421.

Kim, I. H., and G. H. Suh. 2003. Effect of the amount of body condition loss from the dry to near calving periods on the subsequent body condition change, occurrence of postpartum diseases, metabolic parameters and reproductive performance in Holstein dairy cows. Theriogenology 60:1445-1456.

Koves, T. R., J. R. Ussher, R. C. Noland, D. Slentz, M. Mosedale, O. Ilkayeva, J. Bain, R. Stevens, J. R. B. Dyck, C. B. Newgard, G. D. Lopaschuk, and D. M. Muoio. 2008. Mitochondrial overload and incomplete fatty acid oxidation contribute to skeletal muscle insulin resistance. Cell Metab. 7:45-56.

Litherland, N. B., H. M. Dann, and J. K. Drackley. 2011. Prepartum nutrient intake alters palmitate metabolism by liver slices from peripartal dairy cows. J. Dairy Sci. 94:1928-1940. 
McGarry, J. D., and N. F. Brown. 1997. The mitochondrial carnitine palmitoyltransferase system. From concept to molecular analysis. Eur. J. Biochem. 244:1-14.

Mihalik, S. J., B. H. Goodpaster, D. E. Kelley, D. H. Chace, J. Vockley, F. G. Toledo, and J. P. DeLany. 2010. Increased levels of plasma acylcarnitines in obesity and type 2 diabetes and identification of a marker of glucolipotoxicity. Obesity (Silver Spring) 18:1695-1700.

Ospina, P. A., D. V. Nydam, T. Stokol, and T. R. Overton. 2010. Evaluation of nonesterified fatty acids and beta-hydroxybutyrate in transition dairy cattle in the northeastern United States: Critical thresholds for prediction of clinical diseases. J. Dairy Sci. 93:546-554

Peraldi, P., G. S. Hotamisligil, W. A. Buurman, M. F. White, and B. M. Spiegelman. 1996. Tumor necrosis factor (TNF)-alpha inhibits insulin signaling through stimulation of the p55 TNF receptor and activation of sphingomyelinase. J. Biol. Chem. 271:13018-13022.

Raichur, S., S. T. Wang, P. W. Chan, Y. Li, J. Ching, B. Chaurasia, S. Dogra, M. K. Öhman, K. Takeda, S. Sugii, Y. Pewzner-Jung, A. H. Futerman, and S. A. Summers. 2014. CerS2 haploinsufficiency inhibits $\beta$-oxidation and confers susceptibility to diet-induced steatohepatitis and insulin resistance. Cell Metab. 20:687-695.

Ramos-Roman, M. A., L. Sweetman, M. J. Valdez, and E. J. Parks. 2012. Postprandial changes in plasma acylcarnitine concentrations as markers of fatty acid flux in overweight and obesity. Metabolism 61:202-212.

Ribel-Madsen, A., R. Ribel-Madsen, C. Brøns, C. B. Newgard, A. A. Vaag, and L. I. Hellgren. 2016. Plasma acylcarnitine profiling indicates increased fatty acid oxidation relative to tricarboxylic acid cycle capacity in young, healthy low birth weight men. Physiol. Rep. 4:e12977.

Rico, J. E., V. V. R. Bandaru, J. M. Dorskind, N. J. Haughey, and J. W. McFadden. 2015. Plasma ceramides are elevated in overweight Holstein dairy cows experiencing greater lipolysis and insulin resistance during the transition from late pregnancy to early lactation. J. Dairy Sci. 98:7757-7770.

Rico, J. E., A. T. Mathews, J. Lovett, N. J. Haughey, and J. W. McFadden. 2016. Palmitic acid feeding increases ceramide supply in association with increased milk yield, circulating nonesterified fatty acids, and adipose tissue responsiveness to a glucose challenge. J. Dairy Sci. 99:8817-8830.

Rico, J. E., S. Saed-Samii, A. T. Mathews, J. Lovett, N. J. Haughey, and J. W. McFadden. 2017. Temporal changes in sphingolipids and systemic insulin sensitivity during the transition from gestation to lactation. PLoS One 12:e0176787.

Roche, J. R., N. C. Friggens, J. K. Kay, M. W. Fisher, K. J. Stafford and D. P. Berry. 2009. Invited review: Body condition score and its association with dairy cow productivity, health, and welfare. J. Dairy Sci. 92:5769-5801.

Schlegel, G., J. Keller, F. Hirche, S. Geissler, F. J. Schwarz, R. Ringseis, G. I. Stangl, and K. Eder. 2012. Expression of genes involved in hepatic carnitine synthesis and uptake in dairy cows in the transition period and at different stages of lactation. BMC Vet. Res. 8:28.

Schooneman, M. G., F. M. Vaz, S. M. Houten, and M. R. Soeters 2013. Acylcarnitines: Reflecting or inflicting insulin resistance? Diabetes 62:1-8.

Sordillo, L. M., and W. Raphael. 2013. Significance of metabolic stress, lipid mobilization, and inflammation on transition cow disorders. Vet. Clin. North Am. Food Anim. Pract. 29:267-278.

Spiegel, S., and S. Milstien. 2003. Sphingosine-1-phosphate: An enigmatic signaling lipid. Nat. Rev. Mol. Cell Biol. 4:397-407.

Summers, S. A. 2006. Ceramides in insulin resistance and lipotoxicity. Prog. Lipid Res. 45:42-72.

Turpin, S. M., H. T. Nicholls, D. M. Willmes, A. Mourier, S. Brodesser, C. M. Wunderlich, J. Mauer, E. Xu, and P. Hammerschmidt. 2014. Obesity-induced CerS6-dependent C16:0 ceramide production promotes weight gain and glucose intolerance. Cell Metab. 20:678-686.

van Helvoort, A., W. Stoorvogel, G. Van Meer, and K. N. J. Burger. 1997. Sphingomyelin synthase is absent from endosomes. J. Cell Sci. 110:781-788.

Watt, M. J., A. C. Barnett, C. R. Bruce, S. Schenk, J. F. Horowitz, and A. J. Hoy. 2012. Regulation of plasma ceramide levels with fatty acid oversupply: Evidence that the liver detects and secretes de novo synthesised ceramide. Diabetologia 55:2741-2746.

Westerbacka, J., M. Kolak, T. Kiviluoto, P. Arkkila, J. Sirén, A. Hamsten, R. M. Fisher, and H. Yki-Järvinen. 2007. Genes involved in fatty acid partitioning and binding, lipolysis, monocyte/macrophage recruitment, and inflammation are overexpressed in the human fatty liver of insulin-resistant subjects. Diabetes 56:27592765 .

Wildman, E. E., G. M. Jones, P. E. Wagner, and R. L. Bowman. 1982 A dairy cow body condition scoring system and its relationship to selected production characteristics. J. Dairy Sci. 65:495-501.

Xu, C., Z. Wang, R. H. Zhang, H. Y. Zhang, S. X. Fu, and C. Xia. 2011. Effect of NEFA and glucose levels on CPT-I mRNA expression and translation in cultured bovine hepatocytes. J. Vet. Med. Sci. 73:97-101.

Zachut, M., H. Honig, S. Striem, Y. Zick, S. Boura-Halfon, and U. Moallem. 2013. Periparturient dairy cows do not exhibit hepatic insulin resistance, yet adipose-specific insulin resistance occurs in cows prone to high weight loss. J. Dairy Sci. 96:5656-5669. 